Rev. Soc. cient. Parag. 2018;23(2):251-262

ARTÍCULO ORIGINAL / ORIGINAL ARTICLE

\title{
Sedimentos de fondo en los arroyos Tapiracuai y Cuarepotí
}

\section{Bottom sediments from Tapiracuai and Cuarepotí Brooks}

\author{
Franklin D. Flores ${ }^{1}$, Mirna Delgado ${ }^{1}$, Juan F. Facetti Masulli1 ${ }^{1,2}$ \\ ${ }^{1}$ Hydroconsult SRL. Asunción, Paraguay. \\ ${ }^{2}$ Universidad Nacional de Asunción, Facultad de Ingeniería. San Lorenzo, Paraguay. \\ Autor de correspondencia: jfrafama@rieder.net.py \\ DOI: https://doi.org/10.32480/rscp.2018-23-2.251-262 \\ Recibido30/09/2018. Aceptado: 03/12/2018.
}

Resumen: Los sedimentos de fondo de los cuerpos de agua constituyen un reservorio para los cationes metálicos, moléculas y otros productos que a lo largo de lapsos, cortos o largos, pueden constituirse en contaminantes, con efectos deletéreos para la biota; el conocimiento de su contenido es pues muy importante. En este trabajo se investigan en los sedimentos de los arroyos Tapiracuai y Cuarepoti los tenores de elementos de la serie $3 \mathrm{~d}$ que por la presencia/acción de electrones desapareados pueden originar reacciones de radicales libres, así como otros tales como el $\mathrm{Cd}$ y el $\mathrm{Pb}$ de conocida toxicidad. Los valores registrados son más bien bajos. En el examen de sus posibles efectos indeseables, se utilizan con fines de comparación y análisis, los diagramas multielementales (aracnogramas) referidos a los valores recomendados para la corteza superior así como a los valores guías de calidad de sedimentos. Los mismos indican la actual ausencia de riesgo potencial debido a tales elementos; señalan así mismo su utilidad para inferir expeditivamente, la existencia de niveles de efectos indeseables para la biota.

Palabras clave: Sedimentos de fondo, Tapiracuai ,Cuarepoti, contaminantes, serie $3 \mathrm{~d}$, Corteza superior, valores de referencia en sedimentos.

\begin{abstract}
Bottom sediments of water bodies constitute a reservoir for metal cations, molecules and other products that, over a short or long period of time, can become pollutants, with deleterious effects for the biota; the knowledge of its content is therefore very important. In this work, in the sediments of Tapiracuai and Cuarepoti Brooks are investigated elements of the $3 \mathrm{~d}$ series that by the presence / action of unpaired electrons can originate reactions of free radicals, as well as others such as $\mathrm{Cd}$ and $\mathrm{Pb}$ of known toxicity. Their registered values are rather low. In consideration of possible adverse effects were used for comparison and analysis, multielement diagrams (aracnogramas) referred to the recommended values for the upper crust as well as sediments quality guidelines values. They indicate the current lack of potential risk due to such elements; they also show its usefulness to infer expeditiously, the existence of levels of undesirable effects for the biota.
\end{abstract}

Keywords: Bottom sediments, Tapiracuai, Cuarepoti, pollutants, 3d series, Upper Crust, sediments reference values. 


\section{Rev. Soc. cient. Parag. 2018;23(2):251-262}

\section{1.-INTRODUCCIÓN}

El Arroyo Tapiracuai discurre a lo largo de $\sim 82 \mathrm{~km}$ en una cuenca de $19.068 \mathrm{Ha}$. Nace $~$ a 334msnm en Santa Rosa del Mbutuy y descarga a cota $99 \mathrm{msnm}$ en los esteros del Tapiracuai. Es un extenso curso de agua endorreico. El caudal Qm estimado es 11,70 $\mathrm{m}^{3} \mathrm{seg}^{-1}$ y el máximo y mínimo registrados en la referencia (1) fue entre 30 y $6,5 \mathrm{~m}^{3} \mathrm{~s}^{-1}$ respectivamente, en 18 campañas mensuales en un lapso de año y medio. Los estudios de calidad de agua (2) señalaron un $\mathrm{pH}$ promedio de $6,8 \pm 0,22$ unidades y conductividad $33,64 \pm 3,22 \mu \mathrm{S} / \mathrm{cm}$, así como un importante, aunque muy variable contenido de sedimentos en suspensión.

El Cuarepoti se desplaza a lo largo de 61,4 km en una amplia cuenca de $\sim 198.000 \mathrm{Ha}$; su caudal es variable y el curso se amplía en el trecho medio inferior como una planicie con la consiguiente disminución de su velocidad; además está ligado a los niveles del río Paraguay en el área de descarga ya que éste en sus crecidas actúa como freno en el discurrir de aquel. Nace aproximadamente a $275 \mathrm{msnm}$ y descarga en el R Paraguay a cota $68 \mathrm{msnm}$. El caudal Qm estimado es de $5,03 \mathrm{~m}^{3} \mathrm{seg}^{-1}$ y el máximo y el mínimo registrados en la referencia (1) fueron de $\sim 21$ y $0,07 \mathrm{~m}^{3} \mathrm{~s}^{-1}$ respectivamente, en el mismo lapso y en las mismas 18 campañas mencionadas más arriba. Los estudios de calidad de agua (2) señalaron un $\mathrm{pH}$ promedio de 6,96 $\pm 0,29$ unidades y conductividad muy variable $(345,3 \mu \mathrm{S} / \mathrm{cm} \pm 130 \%)$, así como un importante, aunque también muy variable contenido de sedimentos en suspensión.

Los sedimentos, tanto en suspensión como de fondo tienen un prominente papel en la distribución e interacción de los elementos metálicos, mayores, menores y a nivel de trazas, que también tienen su rol en la evolución en/de un cuerpo de agua, la eventual historia del mismo, su "provenance" etc; pero, así como los sedimentos actúan como depósito y sumidero de los metales, constituyen también un componente fundamental del habitat acuático.

Son de interés de este trabajo, los metales tóxicos $\mathrm{Cd}$ y $\mathrm{Pb}$ así como aquellos que a partir de cierta concentraciones pueden disparar efectos eventualmente nocivos para la biota por la presencia/acción de electrones desapareados con la formación de radicales libres como lo son los elementos de la serie $3 \boldsymbol{d}$ del Sistema Periódico.

Entre ellos citamos al Titanio, que es un elemento litófilo refractario incompatible cuyos compuestos en el ciclo geoquímico permanecen en los inatacados $(3,4)$; está generalmente presente en las plantas a bajas concentraciones, muy dependiente de los suelos; podría participar en su metabolismo como un catalizador redox aunque su papel en el desarrollo de las mismas no es bien conocido; al Vanadio con estados de oxidadción III $y$ V. El ión $\mathrm{V}^{3+}$ es un catión de los hidrolizados pero se oxida con facilidad a $\mathrm{V}^{5+}$ que se moviliza fácilmente; se ha demostrado el efecto benéfico de Vanadio sobre el funcionamiento del músculo cardiaco; al Cromo que es un elemento esencial 


\section{Rev. Soc. cient. Parag. 2018;23(2):251-262}

recientemente reconocido como tal (5). Cuando está presente en exceso el cromo produce estrés oxidativo en las células; en la dieta humana la deficiencia en cromo causa

disminución en la tolerancia de la glucosa; por otra parte el cromo incrementa la actividad funcional del sistema inmunológico de los organismos; el $\mathrm{Cr}$ VI es particularmente tóxico; al Manganeso, de varios estados de oxidación: el $\mathrm{Mn}^{2+} \mathrm{se}$ presenta como constituyente normal de enzimas oxidantes. Muy pequeñas cantidades de Mn en las oxidasa y peroxidasa incrementan su capacidad de transportar oxígeno. En el suelo y en sedimentos, la mayor parte del manganeso se halla en los estados de oxidación más altos y solo muy pequeñas cantidades como $\mathrm{Mn}^{2+}$ al Hierro que en los cuerpos de agua superficiales la disolución del $\mathrm{Fe}^{3+}$ depende mucho del $\mathrm{pH}$; a $\mathrm{pH}=1$ o menores el $\mathrm{Fe}^{3+}$ lo hace como aquo complejo; el aumento del $\mathrm{pH}$ tiende a hidrolizar (por fases) el compuesto a las formas más estables (6) como $\left[\mathrm{Fe}\left(\mathrm{H}_{2} \mathrm{O}\right)_{5} \mathrm{OH}\right]^{2+}$ y otras variedades limoníticas, sustancias que precipitan. Muy conocido es el rol del Fe como componente fundamental en la hemoglobina y el transporte de $\mathrm{O}_{2}$; al cobre que se presenta generalmente a nivel de trazas. Se disuelve en forma iónica durante la meteorización incorporándose después a los sedimentos de los hidrolizados. Es un oligoelemento componente de proteínas en enzimas que regulan ciertas reacciones en las plantas que no pueden crecer sin su presencia específica; por lo tanto el cobre tiene un rol esencial en un amplio rango de procesos fisiológicos (por ejemplo formación de clorofila); al Zinc, elemento que si bien su estructura es $\boldsymbol{d}^{\mathbf{1 0}}$, es capaz de catalizar reacciones de radical libre cuando reemplaza a elementos con electrones desapareados. Es cofactor funcional/ regulador de un gran número de enzimas. Es también un nutriente esencial para las plantas. Se disuelve con facilidad durante la meteorización. $(7,8)$.

Entre los estudios de concentración elemental en sedimentos de fondo en cuerpos de agua en el Paraguay pueden citarse aquellos, publicados, realizados en el Lago Ypacarai, Embalse de Itaipú, río Paraguay, Rio Paraná, Embalses de Acaray e Yguazu, rios Carapá, Piratiy, Pilcomayo, Confuso, He'e, Negro, Montelindo, Verde etc (9-16). El presente, ejecutado en estos dos importantes cursos de agua del Departamento de San Pedro el Cuarepotí y el Tapiracuai, tiene por objetivo conocer la concentración de los elementos $\mathrm{Ti}, \mathrm{V}, \mathrm{Cr}, \mathrm{Mn}, \mathrm{Fe}, \mathrm{Cu}, \mathrm{Zn}$ así como $\mathrm{Cd}$ y $\mathrm{Pb}$ en sedimentos de fondo de ambos cursos y estimar su riesgo potencial para la biota.

\section{2.- METODOLOGÍA}

\section{Colecta}

Las muestras fueron colectadas con instrumentos de plástico en triplicado: ellas fueron desecadas al aire luego en estufa a $110^{\circ} \mathrm{C}$, molidas y tamizadas; cada muestra fue preparada por cuarteo.

\section{Análisis}

Los análisis se ejecutaron por espectrofotometría según métodos establecidos (17-19). a) 


\section{Rev. Soc. cient. Parag. 2018;23(2):251-262}

El material $(2,5-5 \mathrm{~g})$ fue disgregado con $\mathrm{K}_{2} \mathrm{CO}_{3}$ o con $\mathrm{KHSO} 4$ en crisol de Pt o tratado con $\mathrm{HNO}_{3}$, según el caso. Luego disuelto y enrasado a volumen conocido.

En una alicuota se precipitan $\mathrm{Ti}$ y $\mathrm{Fe}$ con $\mathrm{NaOH}$; luego se separa el Fe por extracción con éter di-isopropílico y se analiza el Ti como acido peroxititánico a $410 \mathrm{~nm}$ después de tratarlo con $\mathrm{H}_{2} \mathrm{O}_{2}$. En el filtrado proveniente de la precipitación del Ti se determina el V a 560nm después de acidificar con $\mathrm{H}_{2} \mathrm{SO}_{4}$ y tratar con $\mathrm{H}_{2} \mathrm{O}_{2}$. En otra alícuota del material en medio clorhídrico 7,8M, el $\mathrm{Fe}^{3+}$ se extrae con éter di-isopropílico y posteriormente se trata con10-fenantrolina y se determina a $510 \mathrm{~nm}$. El Mn después de ser oxidado con persulfato de $\mathrm{K}$ se determina a $525 \mathrm{~nm}$. El Cu se determina con neocuproina a $457 \mathrm{~nm}$ y el $\mathrm{Zn}$ con ditizona a $535 \mathrm{~nm}$. El Cd y el Pb se determinan con ditizona a 518 y $529 \mathrm{~nm}$. La gestión analítica fue verificada/controlada con el estándar SL1 lake sediments proveniente del IAEA como también con los datos de un estudio paralelo de provenance realizado por Espectrometría de Fluorescencia de Rayos X (20).

\section{3.- RESULTADOS}

En las Tablas 1a y $1 \mathrm{~b}$ se expresan los tenores registrados en arroyos Tapiracuai y Cuarepotí, respectivamente.

Tabla 1 a: Valores registrados en el Arroyo Tapiracuai $\left(\mathrm{mg} \mathrm{kg}^{-1}\right)$

\begin{tabular}{|c|c|c|c|c|c|c|c|c|}
\hline \multirow[t]{2}{*}{ Elementos } & \multicolumn{4}{|c|}{ Tp1 } & \multicolumn{4}{|c|}{ Tp2 } \\
\hline & Prom. & Ds & Max & Min & Prom. & Ds & Max & Min \\
\hline $\mathrm{Ti}$ & 1107 & 302 & 1350 & 590 & 1223 & 280 & 1505 & 760 \\
\hline $\mathrm{V}$ & 20 & 11,6 & 28 & 0 & 24 & 7,5 & 32 & 12 \\
\hline $\mathrm{Cr}$ & ND & & & & & & & \\
\hline $\mathrm{Mn}$ & 102 & 32 & 130 & 49 & 101 & 26 & 127 & 58 \\
\hline $\mathrm{Fe}$ & 1870,7 & 618 & 2380 & 975 & 1694 & 589 & 2289 & 884 \\
\hline $\mathrm{Ni}$ & NA & & & & & & & \\
\hline$\overline{\mathrm{Cu}}$ & 6,4 & 2,2 & 10,2 & 3,9 & 8,3 & 2,8 & 12 & 4,6 \\
\hline $\mathrm{Zn}$ & 30,7 & 18,3 & 48 & 0 & 24,9 & 8,8 & 32 & 10 \\
\hline $\mathrm{Pb}$ & 13,9 & 3,6 & 18 & 8 & 12 & 5,4 & 17,7 & 5,6 \\
\hline $\mathrm{Cd}$ & ND & & & & & & & \\
\hline
\end{tabular}


Rev. Soc. cient. Parag. 2018;23(2):251-262

Tabla 1 b: Valores registrados en el Arroyo Cuarepoti $\left(\mathrm{mg} \mathrm{kg}^{-1}\right)$

\begin{tabular}{|c|c|c|c|c|c|c|c|c|}
\hline & \multicolumn{4}{|c|}{ C1 } & \multicolumn{4}{|c|}{$\mathrm{C2}$} \\
\hline & Prom. & Ds & Max & Min & Prom. & Ds & Max & Min \\
\hline $\mathrm{Ti}$ & 1387 & 493 & 1987 & 620 & 1198 & 348 & 1670 & 590 \\
\hline $\mathrm{V}$ & 17 & 5,4 & 25 & 10 & 20,75 & 12,1 & 30,5 & 0 \\
\hline $\mathrm{Cr}$ & ND & & & & & & & \\
\hline $\mathrm{Mn}$ & 211,7 & 122,3 & 292 & 0 & 156 & 47 & 200,75 & 77 \\
\hline $\mathrm{Fe}$ & 4927,5 & 2583 & 7533 & 1366 & 4530,7 & 2139 & 6010 & 1365 \\
\hline $\mathrm{Ni}$ & NA & & & & & & & \\
\hline $\mathrm{Cu}$ & 18,25 & 6,2 & 25 & 8 & 14,2 & 6,3 & 20,8 & 4 \\
\hline $\mathrm{Zn}$ & 26 & 11 & 37 & 7 & NA & & & \\
\hline $\mathrm{Pb}$ & 10,9 & 3,63 & 15,3 & 5,6 & 13 & 6,3 & 21 & 6 \\
\hline $\mathrm{Cd}$ & ND & & & & & & & \\
\hline
\end{tabular}

En el análisis de los eventuales contaminantes metálicos en los sedimentos en este trabajo se contemplan dos aspectos: a) la abundancia de los elementos en la corteza superior, b) los valores de los elementos estipulados como guías para los sedimentos.

\subsection{Corteza Superior}

A partir de fines del siglo 19, buscando establecer la abundancia de los elementos en la tierra, fueron colectadas y analizadas un gran número de muestras, en particular de la corteza continental. Un excelente resumen de lo logrado hasta la mitad del siglo pasado se puede hallar en la referencia (3). En general los valores correspondientes a la abundancia de los elementos mayores y menores son bastante coincidentes en dichos estudios que también señalaron la limitada información confiable, disponible a esas fechas, respecto de los elementos más raros existentes a nivel de trazas. Esa carencia fue obviada posteriormente por los pioneros trabajos en material sedimentario de diferentes edades, tal como areniscas, shales, calizas etc que mostraron una distribución/padrones muy similar de los elementos de tierras raras en esas variadas muestras aunque diferentes en basaltos, condritos etc $(22,24)$; el análisis y las conclusiones de esos y otros trabajos permitieron la elaboración y publicación de una nueva tabla (25), mejorada y ampliada $(26,29)$. A estas tablas mucho contribuyeron los nuevos métodos analíticos que fueron incorporándose, como el análisis por espectrografía, activación neutrónica, absorción atómica, fluorescencia de Rayos-X, ICP- MS etc. La corteza terrestre presenta dos variantes. Por un lado, la oceánica, de poco espesor $\sim 7 \mathrm{~km}$, que es de edad menor a $200 \mathrm{Ma}$, integrada fundamentalmente por basaltos. Por otro lado, la costra continental, gruesa $\sim 40 \mathrm{~km}$, que contiene prácticamente todas las rocas y minerales observados hasta ahora en la tierra y es de edad entre 4,0-4,4Ga. Así pues, los continentes indican/ testimonian la historia geológica de la evolución de nuestro planeta; el entendimiento de su origen es básico para entender el origen y la diferenciación de la tierra. 


\section{Rev. Soc. cient. Parag. 2018;23(2):251-262}

La costra continental constituye aproximadamente $0,6 \%$ en peso de los silicatos del planeta y contiene una elevada proporción de elementos incompatibles y se extiende verticalmente desde la superficie hasta la discontinuidad de Mohorovic (Moho), que se interpreta como la frontera corteza-manto (25).

A partir de trabajos recientes basados en estudios sísmicos y en la composición de rocas del basamento cristalino y su cubierta sedimentaria, se identifican tres capas. La corteza superior cuyo grosor se estima en $\sim 12-15 \mathrm{Km}$ (30); la corteza media de $\sim 11 \mathrm{Km}$ desde unos $12 \mathrm{~km}$ de la superficie hasta la discontinuidad de Conrad y por fin la corteza inferior desde la discontinuidad de Conrad hasta la Moho que se prolonga por unos $23 \mathrm{~km}$. Así mismo, la corteza se va volviendo más máfica (29).

Por su accesibilidad la corteza superior (UC) ha sido objeto de un gran número de investigaciones y son dos los métodos básicos empleados: promedios pesados de la composición de rocas aflorantes y del promedio de la composición de elementos insolubles/poco solubles (especialmente elementos de energía de estabilización de campo alto (CFSE), de tierras raras etc.), en rocas clásticas sedimentarias de grano fino o los depósitos glaciales. Así se infiere la composición de la costra superior.

Para investigar y comparar la concentración de los elementos en rocas, sedimentos etc se acostumbra a referir/normalizar dicha concentración a los valores recomendados de la corteza superior (UC) construyendo los aracnogramas o diagramas multielementales. El valor unitario obviamente corresponde a los de la corteza superior.

En este trabajo, para tal efecto empleamos las populares tablas de MacLennan (27), utilizadas en anteriores estudios. Los tenores registrados en los sedimentos de fondo de los dos cuerpos de agua normalizados con dichos valores se observan en el aracnograma de la fig 1. En la misma se han incluido para comparación, los valores propuestos como de nivel límite inferior (LEL) que se explica más adelante.

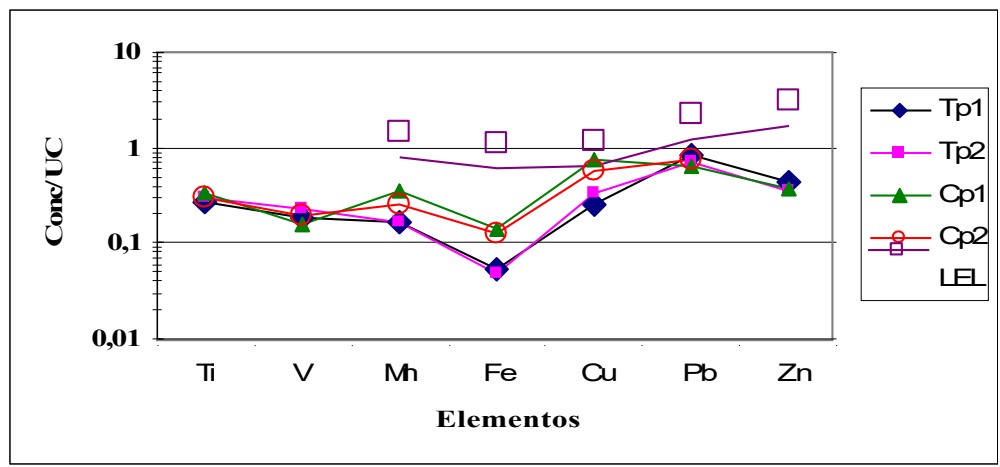

Figura 1: Aracnograma de los valores obtenidos normalizados a los de la UC; Cp Cuarepotí; Tp Tapiracuai; LEL: valores guías de límite inferior (34). 


\section{Rev. Soc. cient. Parag. 2018;23(2):251-262}

Nótese que estos registros normalizados son inferiores a los valores de la corteza superior y que en general, los tenores registrados de estos cationes en los sedimentos de esos arroyos estarían por debajo de los niveles que podrían considerarse de riesgo.

\subsection{Valores Guías en Sedimentos}

Con relación a la estipulación de valores límites en los sedimentos de fondo, ello ha sido y es objeto de algún debate y los tenores que se manejan son de origen básicamente empíricos, aunque debe también mencionarse enfoques teóricos en base a las diferencias en biodisponibilidad estimadas por la partición/distribución en el equilibrio para algunos elementos.

Tradicionalmente la contaminación del sedimento se ha determinado evaluando la concentración de los componentes individuales y con frecuencia comparándolas con una línea de base o background.

De un tiempo a esta parte, se enfoca el tema a la luz de posibles efectos biológicos. Un aspecto muy importante: los sedimentos constituyen una fuente a largo plazo de contaminación de la cadena trófica. Puede darse interrupción en la misma, lo cual podría acarrear la desaparición de tal o cual especie del medio; pueden darse efectos bioacumulativos que afecten los niveles tróficos superiores; pueden darse contaminantes de los sedimentos que por transferencia al medio líquido interfieren la red trófica en la biota acuática etc. El escenario es amplio y complejo y es motivo de numerosas investigaciones, con diversos enfoques referentes a la sanidad de la biota.

Por ello, para elementos/moléculas/productos químicos, los valores límites de referencia se desarrollaron/desarrollan buscando coordinar/emparejar la química de sedimentos con informaciones biológicas ya sean de campo o de laboratorio (por ejemplo, estudios toxicológicos, de biodisponibilidad, etc.) (31).

Con respecto de los contaminantes inorgánicos metálicos los esfuerzos se centran hasta ahora en unos pocos elementos de efectos más o menos conocidos que abarcan buena parte de los $3 \boldsymbol{d}$, y otros como As, $\mathrm{Cd}, \mathrm{Pb}$ etc.

Concomitantemente, fue cambiando la nomenclatura. El usual término "criterio" dio lugar al de "estándar" que tiene significado imperativo, mandatorio, lo que originó resistencias: su alcance no siempre puede extrapolarse ya que su validez podría ser local/puntual solamente. Así mismo, debido a cada situación específica extensa o pequeña, un valor o valores que se encontraren por encima del" límite máximo permitido" podrían también limitar a raja tabla un gran número de acciones u obras de interés psicosocial o inducir a detener alguna obra especifica lo que podría ser erróneo, inoportuno, inconveniente, etc (31). 


\section{Rev. Soc. cient. Parag. 2018;23(2):251-262}

Por lo tanto, se ha sugerido cambiar la terminología de estándar a la de valores guía de calidad de sedimentos (SQG) lo cual tuvo buena aceptación. Y así, en dicho contexto, se han propuestos diversos valores guías con acrónimos del inglés según su nivel de efectos. Así tenemos entre varios otros TEL umbral inferior de efectos; PEL: probable nivel de efectos; LEL efectos de muy bajo nivel; SEL :efectos de nivel severo, ERL efectos de alcance bajo.

También se plantearon tablas de "consenso" provenientes de diferentes enfoques que arrojan valores próximos/similares $(32,33)$ etc. Esos valores propuestos, han dado lugar a la estipulación de las Guías de Calidad de Sedimentos SQG según su acrónimo del inglés. Entre las más citadas están la Canadiense (34), la de la agencia NOAA (35), la flamenca

(32) etc.

Es de interés comparar esos diversos valores guías propuestos con los promedios recomendados de la costra superior para ver qué conclusión se obtiene, recordando que la unidad corresponde al promedio de la Corteza Superior. Para ello, normalizando los valores de algunas pocas* de las series propuestas con respecto a los valores medios de la UC se obtiene la figura $2 \mathrm{a}$.

En la figura $2 b$, se diagraman los valores de consenso también normalizados según los recomendados de la UC.

*evitando desdibujar el correspondiente diagrama multi-elemental con muchas líneas. 


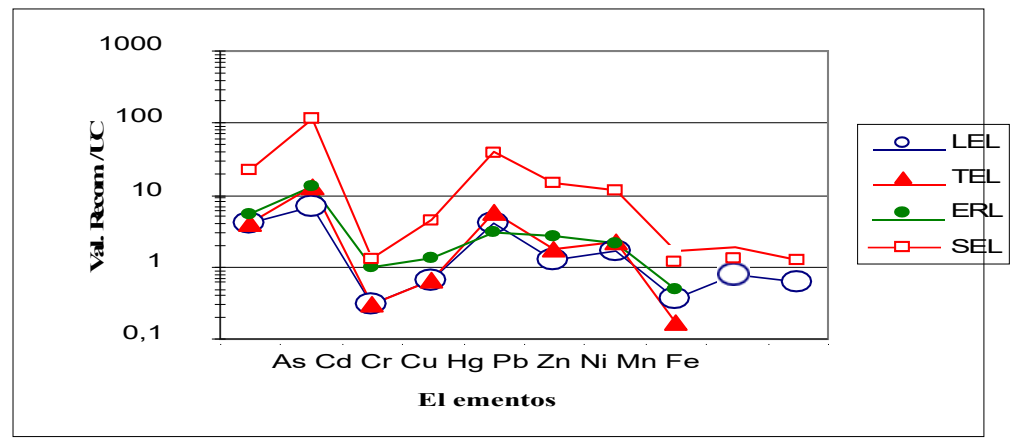

Figura 2a: Aracnogramas de series propuestas; de las referencias $(31,34,35)$ normalizadas según los valores de la UC.

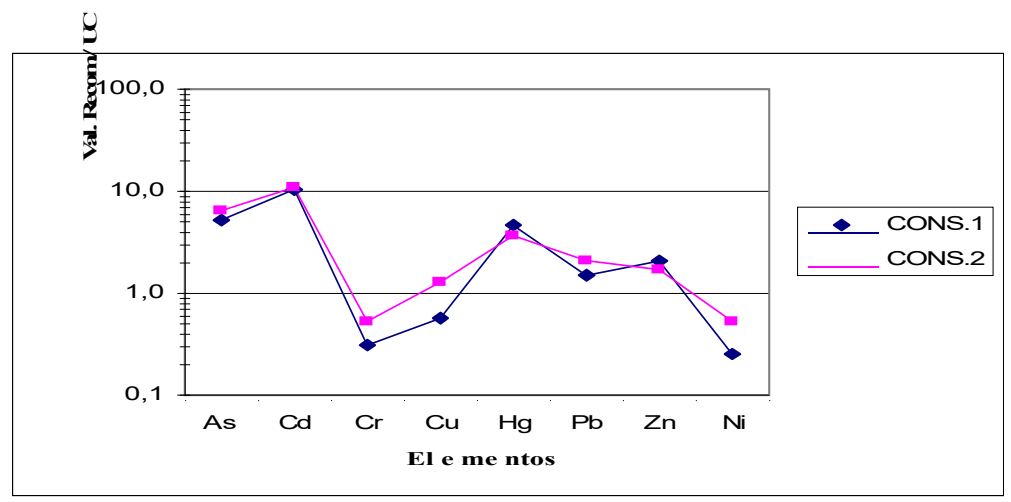

Figura 2b: Aracnogramas de series de consenso propuestas; de las referencias $(32,33)$ normalizados según los valores de la UC.

Las mismas, con relación a los estipulados valores mínimos y de consenso, muestran entre otros, 1) Que algunos de dichos valores mínimos están por encima y otros por debajo de la unidad, esto es, se presentan como de mayor concentración por un lado y de menor por otro que los promedios de la UC. 2) Que el As, Cd, Hg tiene valores promedio UC muy bajos y pequeñas cantidades de sus compuestos en cierto tipo de material pueden superar estos valores: por ejemplo, aquellos que su contenido en los sedimentos de los oxidados puede ser importante como lo es también en el Zn (se acumulan por sorción); 


\section{Rev. Soc. cient. Parag. 2018;23(2):251-262}

el $\mathrm{Pb}$ proviene principalmente del agua del mar y sus evaporados y su contribución es fuertemente antrópica. Por otra parte el Cr permanece en los precipitados de los oxidados $\mathrm{y}$ en los evaporados de modo que los sedimentos casi carecen de Cr: no fue registrado en este trabajo. Así mismo el Ni se distribuye más en la corteza sólida que en los sedimentos (3).

Se deduce que la construcción de los diagramas de tenores analizados de estos elementos, normalizados a los de la UC, permite estimar rápidamente niveles de efectos indeseables. Se trata en ese sentido, de una herramienta muy útil.

Así la figural señala que los elementos acá analizados están por muy debajo de los niveles que podrían considerarse de riesgo para la biota.

\section{4.- CONCLUSIÓN}

El análisis permite concluir que los aracnogramas normalizados a los recomendados de la UC, permiten comparar y estimar fácilmente la existencia o ausencia de niveles de concentración generadores de efectos indeseables. Así mismo que los tenores registrados de estos cationes sugieren que su contenido en los sedimentos de esos arroyos está por debajo de los niveles de concentración que pueden producir efectos deletéreos en la biota.

\section{REFERENCIAS BIBLIOGRÁFICAS}

1. Cáceres F, Delgado M, Parra V, Alonso P. Estudios Hidrológicos en los Ríos Tapiracuai y Cuarepoti. Rev Soc Cient Py. 2016;21:(2):169-180.

2. Flores F, Delgado M, Dávalos A, Facetti MJF. Calidad de agua en los rios Tapiracuai y Cuarepoti- Paraguay Oriental. Rev Soc Cient Paraguay. 2016;21(2);193-206.

3. Rankama K, Sahama Th. Geoquímica (trad. castellana). Madrid: Ed. Aguilar; 1959.

4. Tlustoš P, Cígler P, Hrubý M, Kužel S, Száková J, Balík J. The role of titanium in biomass production and its influence on essential elements contents in field growing crops. Plant Soil Environ. 2005;5:19-25.

5. Vincent JB. The Biochemistry of Chromium. J. Nutr. 2000;130:715-718.

6. Fitzpatrick JA, Facetti MJF. Secchi Disc and Lago de la Republica, Eastern Paraguay. J. Earth Scie. Engin. 2015;5:(8): 482-486.

7. Romero de González V, De Lorenzi A, Kump P, Facetti MJF. Selected Mineral contents in Wheat from Paraguay by EDXRF. J. Chem. Chemical Eng. 2012;6:1114-1120.

8. Kabata-Pendias A. Trace Elements in Soils and Plants. 3rd ed. Boca Ratón: CRC Press; 2000.

9. Delgado M, Lozano F, Facetti Masulli JF. Aspectos limnológicos del Lago YpacaraíEstudios hídricos III. Rev. Soc. Cient. Parag. 2014;19(1):81-93.

10. Romero V, Diaz Z, Kump P, Facetti Masulli JF. Incompatible Elements in Bottom Sediments of the Itaipú Dam Reservoir by EDXRF. J. Radioanal. Nucl. Chem. 2018;316:861- 868.

11. Facetti-Masulli JF, Flores F, Kump P, Geochemical Studies and Elemental Contaminants in the Bay of the City of Asunción. J Chemistry \& Chem Eng. 2013;7:1060-1067. 


\section{Rev. Soc. cient. Parag. 2018;23(2):251-262}

12. Facetti-Masulli JF, Kump P, Romero de González V. Incompatible elements in bottom sediments from the Acaray Dam Eastern Paraguay. Proceeding RAD Conference (in the press). Ohrid; 2018.

13. Facetti Masulli JF, Lozano F, Urbieta A, Delgado M. Calidad de Agua en el Río Carapá. Rev. Soc. Cient. Parag. 2007;12(21):17- 29.

14. Facetti Masulli JF, Lozano F, Urbieta A, Delgado M. Calidad de Agua en el Río Piratiy I. Rev. Soc. Cient. Parag. 2007;12(21):35- 42.

15. Facetti-Masulli JF, Flores F, Dávalos A. Estudios de Calidad de Agua en el río Verde. Rev. Soc. Cient. Parag. 2012;17(1):69-83.

16. Facetti Masulli JF, Kump P. Selected Minor and Trace Elements from Water Bodies of Western Paraguay. J. Radioanal. Nucl. Chem. 2010;286(2):44-51.

17. Hillebrand WF, Lundell GE, Hoffman JI. Applied Inorganic Analysis. 2nd ed. Ne York: J. Wiley \& Sons; 1962.

18. Morrison GH, Freiser H. Solvent extraction in Analytical Chemistry. Ne York: J.Wiley \& Sons; 1957.

19. American Public Health Association. Standard Methods for the Examination of water and wastewater. $19^{\text {th }}$ ed. Washington: United Book Press Inc.; 1995.

20. Kump P, Flores F, Delgado M, Facetti MJF. Trace and minor elements in bottom sediments of selected river and brooks from Eastern Paraguay by X-Ray Fluorescence. En RAD Conference; Herceg Novi, Montenegro; 2019.

21. Alonso P. Soil studies in northern area of Eastern Paraguay. Facultad de Ciencias Agrarias, UNA (en prensa); 2016.

22. Haskin L, Gehl M. The rare earth distribution in sediments. J. Geophys. Res. 1962;67:25372541.

23. Haskin LA, Wildeman TR, Frey FA, Collins KAet al. Rare earths in sediments. J. Geophys. Res. B: Solid Earth .1966:71(24):6091-6105.

24. Schmitt R, Smith R, Lasch J, Mosen A, et al. Abundances of the fourteen rare-earth elements, scandium and yttrium in meteoritic and terrestrial matter. Geochim. Cosmochim. Acta. 1963;27:577-622.

25. Taylor SR. Abundance of chemical elements in the continental crust: a new table Geochim. Cosmochim. Acta. 1964;28:1273-1285.

26. Taylor SR, Mc Lennan SM. The continental crust: its composition and evolution. Carlton: Blackwell Scientific Publication; 1985.

27. Rudnick R, Gao S. Composition of the Continental Crust. Treatise Geochem. New York, Elsevier; 2003.

28. McLennan SM. Relationships between the trace element composition of sedimentary rocks and upper continental crust. Geochem. Geophys. Geosys; 2001;2.

29. Rudnick R, Gao S. The Crust . Treatise of Geochem. $2^{\text {nd }}$ ed. New York: Elsevier Science; 2014.

30. Gao S, Luo TC, Zhang B., Zhang H.-F et al . Chemical composition of the continental crust as revealed by studies in east China. Geochim. Cosmochim Acta. 1998;62:1959-1975.

31. Burton GA. Sediment quality criteria in use around the world. Limnology. 2002;3:65-75 32.

32. Deckere E D, Cooman W D, Leloup V, Meire P et al . Development of sediment quality guidelines for freshwater ecosystems. J Soils and Sedim. 2011;11:504-517.

33. MacDonald DD, Ingersoll G, Berger TA. Development and Evaluation of Consensus-Based Sediment Quality Guidelines for Freshwater Ecosystems. Arch. Environ. Contam. Toxicol. 2000;39:20-31.

34. Canadian Council of Ministers of the Environment (CCME). Protocol for the derivation of 


\section{Rev. Soc. cient. Parag. 2018;23(2):251-262}

Canadian sediment quality guidelines for the protection of aquatic life. Technical Secretariat of the CCME Task Group on Water Quality Guidelines. Otawa; 1995.

35. Long ER, Morgan LG. The Potential for Biological Effects of Sediment-Sorbed Contaminants Tested in the National Status and Trends Program. National Oceanic and Atmospheric Administration. Technical Memorandum. 1991;52. 